ARTICLE

https://doi.org/10.1038/s41467-018-07817-3

\title{
Topological one-way fiber of second Chern number
}

Ling Lu (D) ${ }^{1,2}$, Haozhe Gao ${ }^{1,3}$ \& Zhong Wang (B) ${ }^{4,5}$

One-way waveguides have been discovered as topological edge states in two-dimensional (2D) photonic crystals. Here, we design one-way fiber modes in a 3D magnetic Weyl photonic crystal realizable at microwave frequencies. We first obtain a 3D Chern crystal with a non-zero first Chern number by annihilating the Weyl points through supercell modulation. When the modulation becomes helixes, one-way modes develop along the winding axis, with the number of modes determined by the spatial frequency of the helix. These singlepolarization single-mode and multi-mode one-way fibers, having nearly identical group and phase velocities, are topologically-protected by the second Chern number in the $4 \mathrm{D}$ parameter space of the 3D wavevectors plus the winding angle of the helix. This work suggests a unique way to utilize high-dimensional topological physics using topological defects.

\footnotetext{
${ }^{1}$ Institute of Physics, Chinese Academy of Sciences/Beijing National Laboratory for Condensed Matter Physics, Beijing 100190, China. ${ }^{2}$ Songshan Lake Materials Laboratory, Dongguan, Guangdong 523808, China. ${ }^{3}$ University of Chinese Academy of Sciences, Beijing 100049, China. ${ }^{4}$ Institute for Advanced Study, Tsinghua University, Beijing 100084, China. ${ }^{5}$ Collaborative Innovation Center of Quantum Matter, Beijing 100871, China. Correspondence and requests for materials should be addressed to L.L. (email: linglu@iphy.ac.cn) or to Z.W. (email: wangzhongemail@tsinghua.edu.cn)
} 
T opological photonics ${ }^{1-4}$ started with the realization of oneway edge waveguides ${ }^{5-9}$ as the analog of chiral edge states of the two-dimensional (2D) Chern insulator (or the 2D quantum Hall effect [QHE]), where the number and direction of $1 \mathrm{D}$ edge modes are determined by the $2 \mathrm{D}$ bulk topological invariant: the first Chern number $\left(C_{1}\right)$. Three-dimensional (3D) bands of nonzero $C_{1}$ have also been realized in Weyl photonic crystals ${ }^{10}$, opening doors to $3 \mathrm{D}$ topological phases for photons ${ }^{11,12}$.

Here, we show that, by annihilating a single pair of Weyl points with helix modulations, light can be guided unidirectionally in the core of $3 \mathrm{D}$ photonic crystal fibers (Fig. 1), where the number and direction of one-way modes equal the magnitude and sign of the second Chern number $\left(C_{2}\right)$-the topological invariant of complex vector bundles on $4 \mathrm{D}$ manifolds. This novel approach to create the line-defect states in the $3 \mathrm{D}$ topological bandgap provides a definitive way to obtain arbitrary mode number $\left(C_{2}=-\infty\right.$ to $+\infty$ ) in the one-way fibers by varying the helix frequencies. Furthermore, all the modal dispersions have almost identical group and phase velocities, superior for multimode operations. The same phenomena can be realized in other Weyl systems ${ }^{13-17}$ with time-reversal symmetry breaking.

\section{Results}

Single Weyl dipole. Our starting point is a photonic crystal containing two Weyl points ${ }^{18}$, which were found in the double gyroid (DG) made of magnetic materials. The DG is a minimal surface that can be approximated by the iso-surface of a single triply periodic function: $f(x, y, z)=\sin (2 \pi x / a) \sin (4 \pi y / a) \cos$ $(2 \pi z / a)+\sin (2 \pi y / a) \sin (4 \pi z / a) \cos (2 \pi x / a)+\sin (2 \pi z / a) \sin (4 \pi x / a)$ $\cos (2 \pi y / a)$. This definition, although having a different form, yields almost identical geometry and band structure to those of the DG defined in ref. ${ }^{18}$ by two separate trigonometric functions (one for each gyroid). In Fig. 2a, two cubic cells of the DG are shown, where $f(x, y, z)>f_{0}=0.4$ is filled with gyroelectric material of dielectric constant $\epsilon=\left(\begin{array}{ccc}17 & -6 i & 0 \\ +6 i & 17 & 0 \\ 0 & 0 & 16\end{array}\right)$ and unity magnetic permeability. The rest of the volume is air. In this structure, there exists only two Weyl points (a single "Weyl dipole") separated by about half of the Brillouin zone along $z$ direction, as plotted in Fig. 2b. This means that an infinitesimal supercell modulation of the crystal (in $z$ with a period of 2a) can superimpose the two Weyl points on top of each other to form a 3D Dirac point between four bands ${ }^{11}$ (Fig. 2b), which opens a gap under a finite modulation/coupling strength (Fig. 2d). The fact that a bandgap does not close under small perturbations ensures

a Two-way (single-mode) waveguide
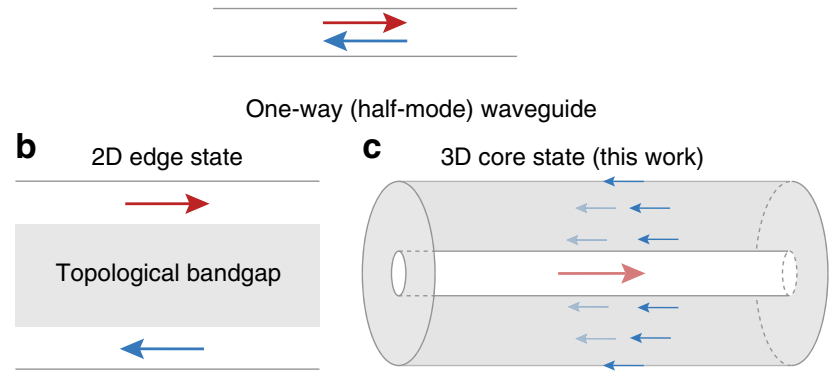

Fig. 1 Conceptual sketch of the one-way (half-mode) waveguides in 2D and 3D. a Single-mode two-way waveguide. b One-way waveguide at the edge of a 2D topological bandgap material, across which the forward and backward modes are spatially separated. c One-way fiber at the core of a 3D topological bandgap material. The backward mode is spatially separated, from the forward core mode, at the outer surface of the fiber cladding the robustness of this approach: certain mismatch between the Weyl-point separation and the wavevector of the modulation can be tolerated.

3D Chern crystal. We create a double-cell periodic modulation along $z$ to annihilate the Weyl points and obtain the 3D Chern crystal, the photonic analog of the 3D Chern insulator (or the 3D QHE) ${ }^{19-21}$. (So far, experimental realization of 3D Chern insulators is only limited to quasi-2D systems ${ }^{22}$ ). This modulation can be implemented in various system parameters, such as volume fraction, refractive index, magnetization, or structural distortion. In this work, we modulate the volume fraction of the DG by modifying the DG equations as follows: $f(x, y, z)>f_{0}+\Delta f \cos$ $(\pi z / a)$, in which $\Delta f=0.07$. The modulated DG is shown in Fig. $2 c$ and its band structure is plotted in Fig. $2 \mathrm{~d}$.

A $3 \mathrm{D}$ Chern crystal is characterized by three first Chern numbers $\mathbf{C}_{\mathbf{1}}=\left(C_{1}^{x}, C_{1}^{y}, C_{1}^{z}\right)$ defined on the $\hat{x}, \hat{y}$, and $\hat{z}$ momentum planes. For example, $C_{1}^{z}$ is defined as

$$
C_{1}^{z} \equiv C_{1}^{z}\left(k_{z}\right)=\frac{1}{2 \pi} \int d^{2} k \operatorname{Tr}\left[\mathcal{F}_{x y}\right]
$$

Because the bulk spectrum is gapped, $C_{1}^{z}$ cannot change as a function of $k_{z}$. When there are $N$ bulk bands below the bandgap, $\mathcal{F}_{x y}$ is an $N \times N$ matrix, whose elements are $\mathcal{F}_{x y}^{\alpha \beta}=\partial_{x} \mathcal{A}_{y}^{\alpha \beta}-\partial_{y} \mathcal{A}_{x}^{\alpha \beta}+i\left[\mathcal{A}_{x}, \mathcal{A}_{y}\right]^{\alpha \beta}$, in which $\alpha, \beta=1,2, \cdots$, $N$. The Berry connection $\mathcal{A}_{i}^{\alpha \beta}(\mathbf{k})=-i\left\langle u^{\alpha}(\mathbf{k})\left|\frac{\partial}{\partial k_{i}}\right| u^{\beta}(\mathbf{k})\right\rangle$, where a

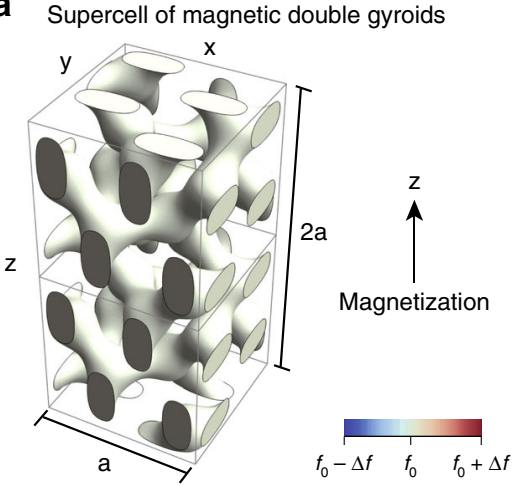

b

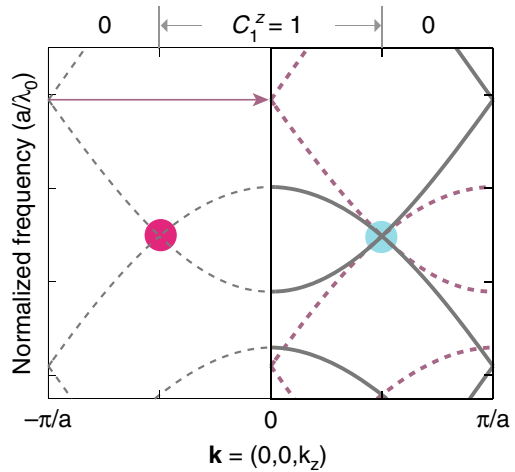

C Plain modulation

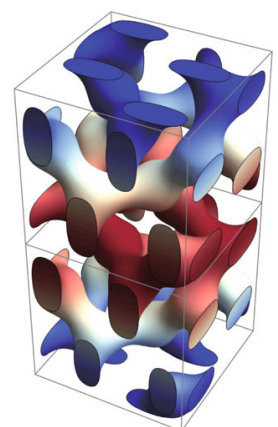

d 3D Chern crystal

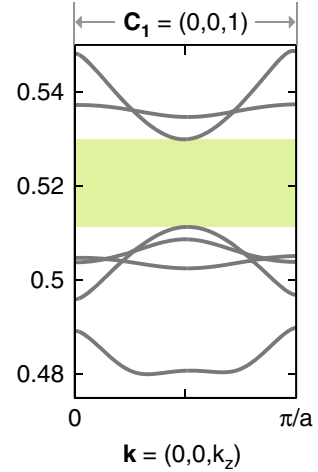

Fig. 2 3D Chern crystal from magnetic Weyl crystals. a Two cubic unit cells of the DG photonic crystal magnetized along $z$. $\mathbf{b}$ The band structure of a cubic cell shows two Weyl points, which fold into one 3D Dirac point in the Brillouin zone of the supercell. c The DG photonic crystal whose volume fraction (blue-red colored) is periodically modulated along $z$. $\mathbf{d}$ The band structure of the 3D Chern crystal whose topological gap frequencies are highlighted in green. $\lambda_{0}$ is the vacuum wavelength 
$\left|u^{\alpha(\beta)}\right\rangle$ are the periodic part of the Bloch wavefunctions (see ref. ${ }^{1}$ for an introduction). Note that the trace of the commutator $\operatorname{Tr}\left[\mathcal{A}_{x}, \mathcal{A}_{y}\right]$ always vanishes for the first Chern class.

The topological invariants of our plainly modulated DG is $\mathbf{C}_{\mathbf{1}}=(0,0,1)$. This can be understood from the original Weyl photonic crystal where $C_{1}^{z}=1$ for half of its Brillouin zone, as illustrated in Fig. 2b. By folding the Brillouin zone to half of its original size, the Chern numbers in different regions add up.

The 3D Chern crystal is a weak topological phase whose weak topological invariants are defined in a lower dimension, as compared with a strong topological phase with a strong topological invariant. It is theoretically known that a lattice dislocation in a weak topological phase creates a 1D topological defect mode ${ }^{23}$. Unfortunately, in our case, a dislocation induces significant lattice distortion that generates many additional nontopological modes in the bandgap.

Fortunately, we propose and demonstrate below that, for a 3D Chern crystal constructed from Weyl crystals, a new approach is available: a smooth helical modulation generates a one-way mode at the core of the helix. The advantage of the helical-modulation approach, compared with the lattice-dislocation approach, is the intactness of the lattice that prevents the generation of nontopological modes in the bandgap. We outline a physical interpretation as follows, and the rigorous calculations are presented in the Methods section. A supercell modulation couples two Weyl points of opposite chiralities, forming a gapped 3D Dirac point with a mass term that is complex-valued. (A 3D Dirac point consists of two Weyl points of opposite chiralities.) Then a helical modulation amounts to a nonzero winding number for the phase of the Dirac mass around the helical axis. It was indicated, in previous theoretical models, that such a topological perturbation can generate topological defect modes in both $2 \mathrm{D}^{24-26}$ and $3 \mathrm{D}$ systems ${ }^{27-30}$.

One-way fiber modes. Now comes the crucial step in our design of topological one-way fibers. Instead of the plain modulation (Fig. 2c), we create a helical modulation by filling the volume satisfying the inequality

$$
f(x, y, z)>f_{0}+\Delta f \cos (\pi z / a+w \theta) .
$$

The modulation now winds as a function of the angle $\theta$ [arctan $(x, y)]$ in the $x-y$ plane, whose spatial frequency is controlled by the signed integer $w$. The sign and magnitude of $w$ determines the direction and number of the one-way modes on the winding axes. This is illustrated in the upper panels of Fig. 3 for $w=+1,+2$, +3 , corresponding to single, double, and triple helix one-way fibers.

The band structures of the one-way fibers are shown in Fig. 3f, i, 1 . They were calculated using MIT Photonic Bands on a $11 a \times 11 a \times 2 a$ cubic supercell. The spectra exhibit one-way modes within the bulk bandgap. The fields of the topological fiber modes are localized around the helix cores (Fig. 3g, j, m), and the localization length is minimized at the mid-gap frequencies. In general, the higher-order mode profiles are more extended in the real space. For the multimode fields of $w=+2$, +3 , instead of the mid-gap frequencies, we plot the mode profiles close to the band-edge frequencies. Because multimode dispersions are almost degenerate at the middle of the bandgap (Fig. 3i, 1), it is difficult to resolve their intrinsic mode patterns from their linear superpositions.

In Fig. 3f, i, l, all one-way-fiber dispersions (green lines) have very similar phase and group velocities. In the multimode cases, their dispersions are almost degenerate at the mid-gap frequencies. This is due to the fact that these defect modes originated from the same Weyl bulk bands, so they all share the same Brillouin-zone location and group velocities as those of the original Weyl cones. This behavior is different from that of the multimode one-way edge waveguides in $2 \mathrm{D}^{9}$, where the edge modal dispersions have different phase or group velocities. This can be attributed to the fact that the edge environment, of sharp terminations, is distinct from the environment of the $2 \mathrm{D}$ bulk lattice. While, here, there are no sharp interfaces in the 3D oneway fibers. This unique feature, of multiple fiber modes having almost identical dispersions, ensures that multimode signals propagate at the same speed for both energy and phase.

Time-domain simulations. To visualize and confirm our prediction made by spectral dispersions, we simulate the wave dynamics of the one-way fiber $(w=+1)$ in the real space in Fig. 4. Due to the huge computation domain, the finite-difference time-domain (FDTD) method is adopted for its nice scaling with the computation size. We use the commercial software EastWave $^{31}$ for its capability in handling nonreciprocal materials.

In Fig. 4, we compare the one-way fiber to a regular fiber having a core diameter of $2 a$ with a dielectric constant of 16 in air. In both cases, the computation domain is $20 a \times 20 a \times 26.5 a$, in $(x, y, z)$ directions, and the mesh resolution is a/30. The perfectly matched layers (PMLs) are used at all six boundary planes. A dipole source polarized in $z$ direction was placed at the position $(0.1,0.2,5.5) a$ to excite the fiber mode. A metallic ball of diameter $1.5 a$ is placed at $(0,0,14.5) a$ to test the robustness of the mode. Obviously, the one-way mode perfectly circumvents the metal sphere without any scattering losses, while the regular fiber mode backscatters. We also note that fiber bends, disrupting the 3D bandgap of cladding, can cause photon loss.

Second Chern number. It is natural to ask for a topological invariant for the one-way fibers. With the simplest helix modulation of the form of Eq. (2), it is intuitive to guess that $w$ is the topological invariant, since the number and direction of the oneway modes match the magnitude and sign of $w$. However, this observation does not work if we consider the modulation of the general form as $f(x, y, z)>f_{0}+\sum_{w} h_{w} \cos (\pi z / a+w \theta)$, where $h_{w}$ are real-valued constants.

For a lattice dislocation in a 3D Chern crystal, it is known ${ }^{32}$ that the number of chiral modes is given by $\mathbf{C}_{1} \cdot \mathbf{b}$, where the dimensionless Burgers vector (b) represents the magnitude and direction of the lattice distortion. However, this approach cannot be applied to our system due to the lack of a unique "Burgers vector" other than that in the simplest case (as of Eq. (2)).

We show that the formal topological invariant of our one-way fibers is the second Chern number $\left(C_{2}\right)$, the strong topological invariant in our system. Note that, far away from the axis of the helix, the Bloch Hamiltonian smoothly varies with $\theta$ and is a smooth function of the four variables $\left(k_{x}, k_{y}, k_{z}, \theta\right)$. Since $\left(k_{x}, k_{y}\right.$, $\left.k_{z}, \theta\right)$ span a four-dimensional parameter space with periodic boundary conditions (a $4 \mathrm{D}$ torus), the second Chern number ${ }^{32,33}$ can be defined:

$$
C_{2}=\frac{1}{4 \pi^{2}} \int d^{3} k d \theta \operatorname{Tr}\left[\mathcal{F}_{x y} \mathcal{F}_{z \theta}+\mathcal{F}_{y z} \mathcal{F}_{x \theta}+\mathcal{F}_{z x} \mathcal{F}_{y \theta}\right] .
$$

Similar to the definitions in Eq. (1), $\mathcal{F}_{i j}^{\alpha \beta}=\partial_{i} \mathcal{A}_{j}^{\alpha \beta}-\partial_{j} \mathcal{A}_{i}^{\alpha \beta}+i\left[\mathcal{A}_{i}, \mathcal{A}_{j}\right]^{\alpha \beta}$, in which $\alpha, \quad \beta$ are the band indices. The non-Abelian Berry potential $\mathcal{A}_{i}^{\alpha \beta}(\mathbf{k}, \theta)=-i\left\langle u^{\alpha}(\mathbf{k}, \theta)\left|\frac{\partial}{\partial k_{i}}\right| u^{\beta}(\mathbf{k}, \theta)\right\rangle$, where $\left|u^{\alpha(\beta)}\right\rangle$ are the eigenfunctions and $k_{i}$ runs through $k_{x}, k_{y}, k_{z}$, $\theta$. It is notable that this definition of $C_{2}$ involves three variables $\left(k_{x, y, z}\right)$ in the reciprocal space and one variable $(\theta)$ in the real space, in contrary to the four momentum variables in the $4 \mathrm{D} \mathrm{QHE}^{33-40}$. 
a
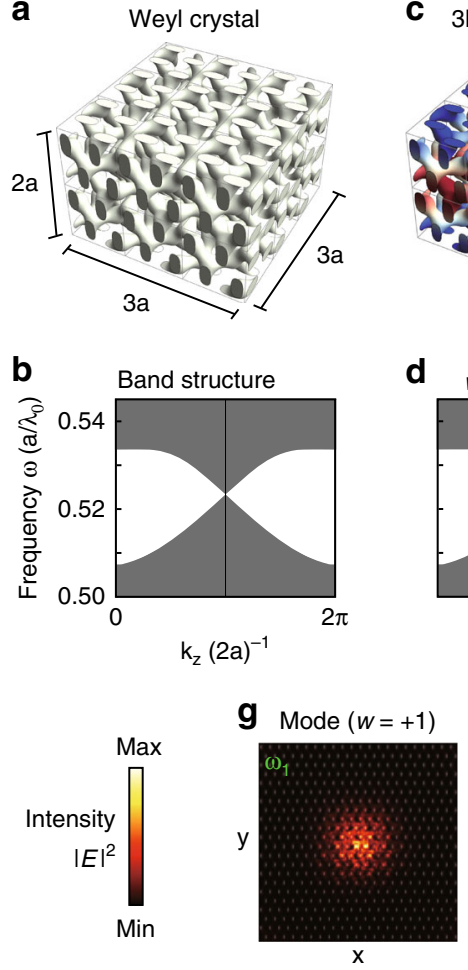

C 3D Chern crystal

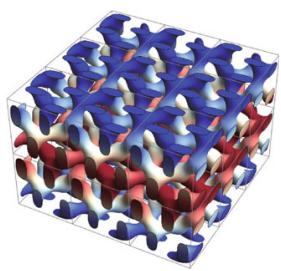

d

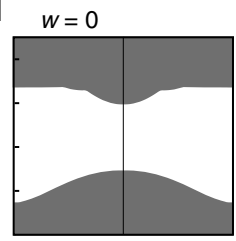

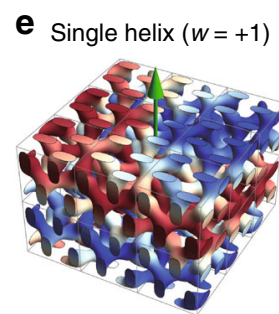

$\mathbf{f}$

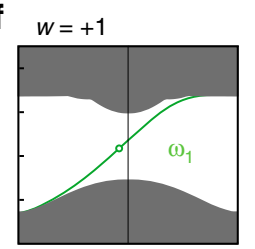

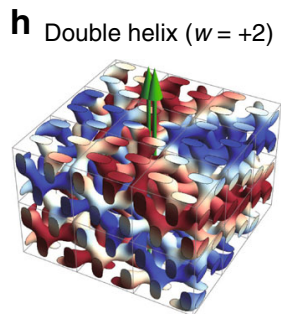

i $w=+2$

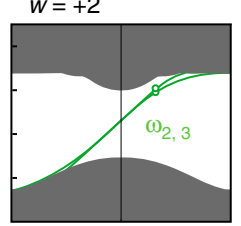

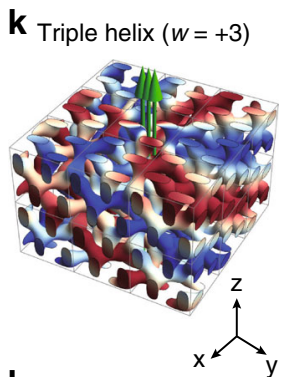

I $w=+3$

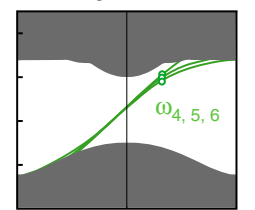

j

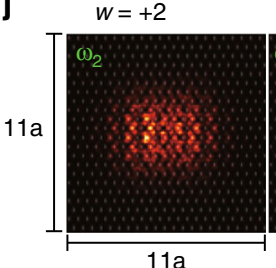

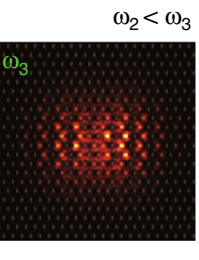
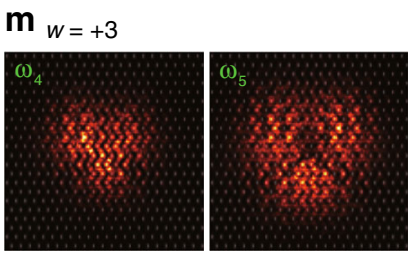

Fig. 3 Single and multimode fibers in helically modulated magnetic DGs. a The DG structure without modulation is shown in a $3 \times 3 \times 2$ cubic cell and its projected Weyl band structure is shown in $\mathbf{b}$. $\mathbf{c}$ The DG structure of a plain modulation $(w=0)$, and its projected gapped band structure is shown in $\mathbf{d}$. $\mathbf{e}$ The single helix DG structure $(w=+1)$, whose helix center supports one one-way fiber mode. The fiber dispersion and mode profile are shown in $\mathbf{f}, \mathbf{g}$. $\mathbf{h}$ The double helix DG structure $(w=+2)$, whose helix center supports two one-way fiber modes. The fiber dispersions and mode profiles are shown in $\mathbf{i}, \mathbf{j}$. $\mathbf{k}$ The triple helix DG structure $(w=+3)$, whose helix center supports three one-way fiber modes. The fiber dispersions and mode profiles are shown in $\mathbf{I}, \mathbf{m}$

a

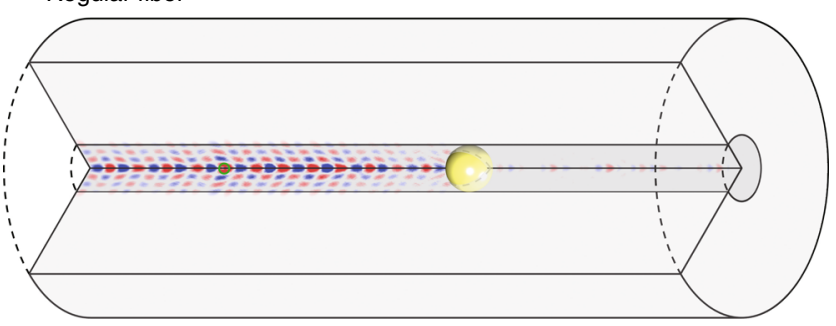

b One-way fiber

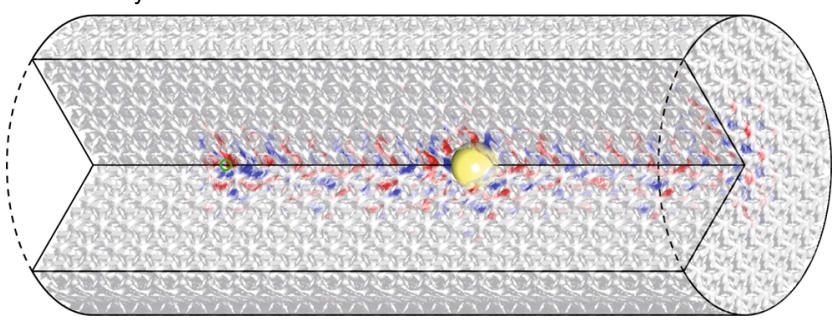

Fig. 4 Comparison of a regular fiber and the one-way fiber. a A regular twoway fiber mode with strong backscattering off the metallic ball (refer to Supplementary Movie 1). b The one-way fiber mode $(w=+1)$ has no backscattering (refer to Supplementary Movie 2). The magnetic gyroid photonic-crystal structure is plotted in gray. One-quarter of the fiber volume was removed to expose the electric field of the fiber mode excited by the continuous point source placed at the green circle
Consequently, the Berry curvature $\mathcal{F}_{i \theta}$ is even while $\mathcal{F}_{i j}$ is odd under time reversal, where $i$ or $j$ represents one of $x, y$, and $z$. Although in $4 \mathrm{D}$ QHE, $C_{2}$ can be nonzero without breaking timereversal symmetry, nonzero $C_{2}$ requires time-reversal breaking in our system, which is consistent with the one-way phenomena.

In the Methods section, we carry out the explicit calculations of $\mathrm{C}_{2}$, which is consistent with our numerical findings in Fig. 3. The topological protection by the second Chern number indicates that the physical origin of the one-way fiber modes is fundamentally different from that of the edge modes of the 2D Chern crystals ${ }^{5,6}$ (2D Chern insulator or 2D QHE), whose topology is captured by the first Chern number. We note that, although in our system both the weak indices $\left(\mathbf{C}_{1}\right)$ and the strong index $\left(C_{2}\right)$ are nonzero, it is possible to construct a one-way fiber design with zero $\mathbf{C}_{\mathbf{1}}$ and nonzero $C_{2}$. For example, when the separation between the two Weyl points shrinks to zero (forming a 3D Dirac point), one can apply only angular $(\theta)$ modulations to obtain a one-way fiber of nonzero $C_{2}$ but zero $\mathbf{C}_{\mathbf{1}}$.

\section{Discussion}

Experimentally, one-way fibers can be constructed using gyromagnetic materials $6,9,41$ at microwave frequencies. For higher frequencies, there lacks magnetic materials with high Verdet constants and low loss. Nevertheless, we discuss the potential relevant technologies below. Toward optical frequencies, there is progress on magnetic fibers ${ }^{42,43}$ and gyroelectric materials ${ }^{44,45}$. The opt-acoustic coupling 46 in fibers provides another possibility for breaking time-reversal symmetry. A DG fiber can either be made by drawing a $3 \mathrm{D}$-printed preform or potentially by 
self-assembly ${ }^{47,48}$ during the drawing process. Three-dimensional direct writing ${ }^{49}$ and interference lithography ${ }^{50}$ can also be adopted. Finally, the chiral modulation can be created by spinning the fiber during drawing, as demonstrated in the chiral fibers ${ }^{51,52}$.

The proposal of one-way fibers enriches the prospects of device applications for the Weyl materials and topological photonics. It also brings a new playground for the realization of higherdimensional topological physics ${ }^{53,54}$. Topological fibers could also inspire new directions, design principles for novel fibers ${ }^{55-57}$.

\section{Methods}

Effective 3D Dirac Hamiltonian. In the main text, we have presented our design of the one-way fiber and the results of band-structure calculations. To gain a simple analytical understanding of the one-way modes, we outline below an effective Hamiltonian description, using the low-energy Dirac Hamiltonian. The picture can be summarized as follows. In terms of the effective Dirac Hamiltonian, the modulation corresponds to the presence of a Dirac mass. The helix modulation introduces a topologically nontrivial configuration of the Dirac mass (nonzero winding number of the phase of Dirac mass), which creates topologically one-way defect modes.

In the absence of modulations, the double-gyroid crystal hosts two Weyl points with opposite chirality, either of which is described by a $2 \times 2$ effective Weyl Hamiltonian. We can combine them as a $4 \times 4$ block-diagonal Dirac Hamiltonan: $H_{\mathrm{D}}=-i v\left(\sigma_{x} \partial_{x}+\sigma_{y} \partial_{y}+\sigma_{z} \partial_{z}\right) \tau_{z}$ where $\sigma_{i}, \tau_{i}(i=x, y, z)$ are Pauli matrices, $v$ is the group velocity (for simplicity, we take isotropic group velocities with $v>0$ ). As we have seen in the main text, a modulation with periodicity $2 a$ in the $z$ direction gaps out the Weyl points. In the effective Dirac-Hamiltonian description, the frequency gap is due to the Dirac mass terms. There are only two possible Dirac mass terms: $m_{1} \tau_{x}$ and $m_{2} \tau_{y}$, both of which anti-commute with the $\sigma_{i} \tau_{z}$ terms in $H_{\mathrm{D}}$. It is thus expected that the modulation amounts to the presence of these Dirac mass terms in the low-energy effective Hamiltonian. In general, both $m_{1}$ and $m_{2}$ can be nonzero, and the mass term can be rewritten as $m_{1} \tau_{x}+m_{2} \tau_{y}=m \tau_{+}+m^{*} \tau_{-}$, with $\tau_{ \pm} \equiv\left(\tau_{x} \pm i \tau_{y}\right) / 2$ and $m \equiv m_{1}-i m_{2}$. The full effective Hamiltonian, with the effect of modulation included as the Dirac mass, can be written as

$$
H_{\text {eff }}=-i v\left(\sigma_{x} \partial_{x}+\sigma_{y} \partial_{y}+\sigma_{z} \partial_{z}\right) \tau_{z}+m \tau_{+}+m^{*} \tau_{-},
$$

from which we can readily see that a frequency gap $2|m|$ is generated by the Dirac mass terms.

In this effective Hamiltonian, the chirality flipping terms containing $\tau_{ \pm}$couple the states near the two Weyl points; therefore, they should come from the modulation. Suppose that the modulation can be modeled in the effective potential $V(\mathbf{r})=V_{\mathbf{Q}} \exp (+i \mathbf{Q} \cdot \mathbf{r})+V_{\mathbf{Q}}^{\dagger} \exp (-i \mathbf{Q} \cdot \mathbf{r})+\cdots$, where $\mathbf{Q}=(0,0, \pi / a)$ is the wavevector that couples the two Weyl points. We can see that $\exp ( \pm i \mathbf{Q} \cdot \boldsymbol{r}) \rightarrow \tau_{ \pm}$is valid near the Weyl points, and we have $m=V_{\mathbf{Q}}$, in other words, the complexvalued Dirac mass is simply the Q-component of the perturbation.

Now, we show that the displacement of modulation (d) amounts to the phase change of the Dirac mass $(m)$. If we displace the modulation by a distance $\mathbf{d}$, then the perturbation becomes $V(\mathbf{r}+\mathbf{d})$, which can be expanded as $V(\mathbf{r}+\mathbf{d})$ $=V_{\mathbf{Q}} \exp [i \mathbf{Q} \cdot(\mathbf{r}+\mathbf{d})]+V_{\mathbf{Q}}^{\dagger} \exp [-i \mathbf{Q} \cdot(\mathbf{r}+\mathbf{d})]+\cdots$, thus we can see that the displacement causes $V_{\mathbf{Q}} \rightarrow V_{\mathbf{Q}} \exp (i \mathbf{Q} \cdot \mathbf{d})$, or equivalently, $m \rightarrow m \exp (i \mathbf{Q} \cdot \mathbf{d})$. For the helix-shape modulation along the axis $r=0$, as described in the main text, the displacement $\mathbf{d}$ is a function of $\theta$ such that $\mathbf{Q} \cdot \mathbf{d}=w \theta$. Here, we use the cylindrical coordinates $(x, y, z) \equiv(r \cos \theta, r \sin \theta, z)$. Therefore, we have a nonzero winding of the phase of Dirac mass around the axis, namely, $m(\theta)=m_{0} \exp (i w \theta)$, in which $m_{0} \equiv$ $m(\theta=0)$. The overall phase of $m_{0}$ can be changed by rotating the coordinate systems around the $r=0$ axis, thus we are free to take $m_{0}$ to be real-valued and positive.

Analytic solutions of one-way modes. For the effective 3D Dirac Hamiltonian with a nonzero winding of the phase of Dirac mass (with winding number $w$ ), which is a consequence of the helix perturbation, we shall show that there exist $|w|$ topological one-way modes. We can rewrite Eq. (4) in the cylindrical coordinates as

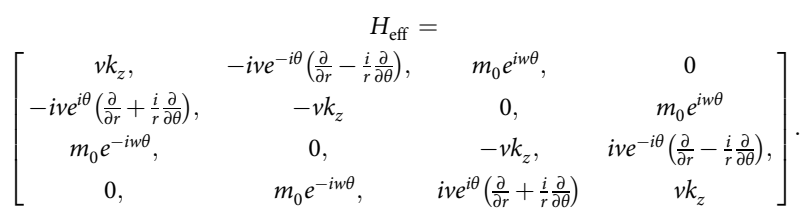

where we have taken advantage of the translational symmetry in the $z$ direction by replacing $-i \partial_{z}$ by $k_{z}$. For notational simplicity, we shall keep implicit the common factor $\exp \left(i k_{z} z\right)$ in the eigenfunction. For a reason that will become clear shortly, we look for eigenfunctions of the form of $\psi=\left[\psi_{1}, 0,0, \psi_{4}\right]^{T}$. The eigenvalues are
$E=v k_{z}$, and the eigenfunctions satisfy

$$
\begin{aligned}
& -i v e^{i \theta}\left(\frac{\partial}{\partial r}+\frac{i}{r} \frac{\partial}{\partial \theta}\right) \psi_{1}+m_{0} e^{i w \theta} \psi_{4}=0, \\
& m_{0} e^{-i w \theta} \psi_{1}+i v e^{-i \theta}\left(\frac{\partial}{\partial r}-\frac{i}{r} \frac{\partial}{\partial \theta}\right) \psi_{4}=0 .
\end{aligned}
$$

It is not difficult to observe that the second equation is equivalent to the first one if we take $\psi_{4}= \pm \psi_{1}^{*}$. Let us focus on the $\psi_{4}=+\psi_{1}^{*}$ case first. With this condition, the above two equations are reduced to a single equation

$$
-i v e^{i \theta}\left(\frac{\partial}{\partial r}+\frac{i}{r} \frac{\partial}{\partial \theta}\right) \psi_{1}+m_{0} e^{i w \theta} \psi_{1}^{*}=0
$$

For the $w=+1$ case, the common $\exp (i \theta)$ factors can be eliminated; thus, the equation becomes especially simple, and the solution can be found analytically as

$$
\left|\psi_{w=+1}\right\rangle=\left(\begin{array}{c}
e^{i \pi / 4} \\
0 \\
0 \\
e^{-i \pi / 4}
\end{array}\right) e^{-\frac{m_{0}}{v}} .
$$

For an arbitrary integer $w \geq+1$, by analysis analogous to ref. ${ }^{24}$, we can show that there exist $w$ localized modes. In fact, we can take the following ansatz for Eq. (7):

$$
\psi_{1}^{(l)}=e^{i \pi / 4}\left[u_{l} e^{i l \theta}+v_{l} e^{i(w-1-l) \theta}\right],
$$

with an integer parameter $l$, whose acceptable values are to be determined. We first notice that when $l=(w-1) / 2, e^{i l \theta}$ and $e^{i(w-1-l) \theta}$ are actually equal, and the $v_{l}$ term is redundant. Let us first focus on the cases $l \neq(w-1) / 2$. The special case $l=(w-$ 1)/2, with $e^{i l \theta}=e^{i(w-1-l) \theta}$, will be discussed separately later.

According to Eq. (7), the coefficient functions $u_{l}, v_{l}$ have to satisfy

$$
\begin{gathered}
v\left(\frac{\partial}{\partial r}-\frac{l}{r}\right) u_{l}+m_{0} v_{l}=0 \\
v\left(\frac{\partial}{\partial r}-\frac{w-1-l}{r}\right) v_{l}+m_{0} u_{l}=0 .
\end{gathered}
$$

The asymptotic behaviors of $u_{b}, v_{l}$ in the $r \rightarrow 0$ limit can be found as (I) $u_{l}: r^{l}, v_{l} \sim r^{l+1}$ or (II) $u_{l}: r^{w-l}, v_{l}: r^{w-l-1}$. On the other hand, the asymptotic behaviors in the $r \rightarrow \infty$ limit are (a) $u_{l} \rightarrow \exp \left(-\frac{m_{0}}{v} r\right), v_{l} \rightarrow \exp \left(-\frac{m_{0}}{v} r\right)$ or (b) $u_{l} \rightarrow \exp \left(\frac{m_{0}}{v} r\right), v_{l} \rightarrow \exp \left(\frac{m_{0}}{v} r\right)$, only the first of which is normalizable in the $r \rightarrow \infty$ regime. A normalizable solution must have behavior (a) in the $r \rightarrow \infty$ limit, which is generally a superposition of (I) and (II) in the $r \rightarrow 0$ regime. Therefore, the normalizability of the solution in the $r \rightarrow 0$ limit requires that both (I) and (II) are normalizable, which leads to the constraint

$$
0 \leq l \leq w-1 \text {. }
$$

Thus, we have proved that, leaving out the special case $l \neq(w-1) / 2$ undetermined, there exists one normalizable solution for every integer $l=0,1,2, \cdots, w-1$.

However, the solutions with $l>(w-1) / 2$ are redundant, because the solutions for $l$ and $l^{\prime}$ with $l+l^{\prime}=w-1$ are actually the same one, as can be appreciated from Eq. (9). Therefore, the total number of solutions with $\psi_{4}=+\psi_{1}^{*}$ is the number of a nonnegative integer smaller than $(w-1) / 2$, which is $[w / 2]$ (here, "[ $\cdots]$ " denotes the floor function, mapping a real number to the largest previous integer), excluding a possible solution with $l=(w-1) / 2$.

Now we consider the other choice: $\psi_{4}=-\psi_{1}^{*}$. By calculations similar to the case $\psi_{4}=\psi_{1}^{*}$, we can obtain equations similar to Eq. (7), except that the "+" sign before $m_{0}$ is replaced by "-". We adopt the same ansatz as given in Eq. (9), and follows the steps below Eq. (9), solving the case $l \neq(w-1) / 2$ first. It is found that the number of solutions is $[w / 2]$.

Finally, we study the special case $l=(w-1) / 2$ (this case needs consideration only when $w$ is odd; for $w$ even, this option is automatically absent). Given this value of $l$, the second term in Eq. (9) becomes redundant, thus we can take

$$
\psi_{1}^{(l)}=e^{i \pi / 4} u_{l} e^{i l \theta} .
$$

Under the choice $\psi_{4}= \pm \psi_{1}^{*}$, we obtain the single differential equation

$$
v\left(\frac{\partial}{\partial r}-\frac{l}{r}\right) u_{l} \pm m_{0} u_{l}=0 .
$$

For the choice " + " of the " \pm ", Eq. (13) has a single normalizable solution with asymptotic behaviors $u_{l} \rightarrow r^{l}$ in the $r \rightarrow 0$ limit and $u_{l} \rightarrow \exp \left(-\frac{m_{0}}{y} r\right)$ in the $r \rightarrow \infty$ limit. For the choice "-" of the " \pm ", Eq. (13) leads to $u_{l} \rightarrow \exp \left(\frac{m_{0}}{v} r\right)$ in the $r \rightarrow \infty$ limit, which is apparently not normalizable. Therefore, there exists a single normalizable localized mode, in the $\psi_{4}=+\psi_{1}^{*}$ sector, for the special case $l=(w-$ 1)/2. We also note that, if we take $m_{0}<0$ instead of $m_{0}>0$, the normalizable solution would be present in the $\psi_{4}=-\psi_{1}^{*}$ sector but absent in the $\psi_{4}=+\psi_{1}^{*}$ sector, thus the total number of solution is the same.

Let us summarize the above calculations as follows. When $w$ is odd, the total number of normalizable solutions is $2[w / 2]+1=w$; when $w$ is even, the total number of normalizable solutions is $2[w / 2]=w$. Therefore, the total number of topological modes is always $w$, irrespective of the parity (odd/even) of $w$. Furthermore, our calculation shows that the eigenvalues take the simple 
form

$$
E\left(k_{z}\right)=+v k_{z},
$$

Thus, all these $w$ modes propagate along the $+z$ direction, with the same velocity $v$. Finally, let us discuss the one-way modes for the integer $w<0$. A solution of the form of $\psi=\left[\psi_{1}, 0,0, \psi_{4}\right]^{T}$ does not exist in this case, because the condition given in Eq. (11) can never be satisfied. On the other hand, solutions of the form of $\psi=[0$, $\left.\psi_{2}, \psi_{3}, 0\right]^{T}$ can be found. In fact, we can follow the steps above and obtain the equations

$$
\begin{gathered}
-i v e^{-i \theta}\left(\frac{\partial}{\partial r}-\frac{i}{r} \frac{\partial}{\partial \theta}\right) \psi_{2}+m_{0} e^{i w \theta} \psi_{3}=0 \\
m_{0} e^{-i w \theta} \psi_{2}+i v e^{i \theta}\left(\frac{\partial}{\partial r}+\frac{i}{r} \frac{\partial}{\partial \theta}\right) \psi_{3}=0,
\end{gathered}
$$

whose complex conjugations are

$$
\begin{aligned}
& -i v e^{i \theta}\left(\frac{\partial}{\partial r}+\frac{i}{r} \frac{\partial}{\partial \theta}\right) \psi_{2}+m_{0} e^{i|w| \theta}\left(-\psi_{3}\right)=0, \\
& m_{0} e^{-i|w| \theta} \psi_{2}+i v e^{-i \theta}\left(\frac{\partial}{\partial r}-\frac{i}{r} \frac{\partial}{\partial \theta}\right)\left(-\psi_{3}\right)=0 .
\end{aligned}
$$

We can see that Eq. (16) is the same as Eq. (6) except that $\psi_{2}$ and $-\psi_{3}$ take the place of $\psi_{1}$ and $\psi_{4}$, respectively. Now, our previous analysis for Eq. (6) with $w \geq+1$ immediately tells us that the number of one-way modes for $w<0$ is $|w|$. Because the solutions for $w<0$ take the form of $\psi=\left[0, \psi_{2}, \psi_{3}, 0\right]^{T}$, the dispersion is $E\left(k_{z}\right)=$ $-v k_{z}$, thus all the one-way modes propagate in the $-z$ direction.

Calculation of the second Chern number $\boldsymbol{C}_{\mathbf{2}}$. The effective Hamiltonian Eq. (4) takes the form of

$$
H_{\text {eff }}=\sum_{a=1}^{5} d_{a} \Gamma^{a}
$$

where the Dirac matrices $\Gamma^{a}=\sigma_{a} \tau_{z}(a=1,2,3), \Gamma^{4}=\tau_{x}, \Gamma^{5}=\tau_{y}$, the coefficient functions $d_{a}=v k_{a}(a=1,2,3), d_{4}=\operatorname{Re}(m), d_{5}=-\operatorname{Im}(m)$. A straightforward calculation of $C_{2}$, as in ref. ${ }^{33}$, leads to

$$
\begin{gathered}
C_{2}=\frac{3}{8 \pi^{2}} \int d \theta d^{3} k \epsilon^{a b c d e} \hat{d}_{a} \frac{\partial \hat{d}_{b}}{\partial k_{x}} \frac{\partial \hat{d}_{c}}{\partial k_{y}} \frac{\partial \hat{d}_{d}}{\partial k_{z}} \frac{\partial \hat{d}_{e}}{\partial \theta} \\
=\frac{1}{2 \pi} \int_{0}^{2 \pi} d \theta \frac{d[\arg (m(\theta))]}{d \theta},
\end{gathered}
$$

where $\hat{d}_{a}=d_{a} / \sqrt{\sum_{b=1}^{5} d_{b}^{2}}$. With the Dirac mass $m=m_{0} \exp (i w \theta)$, we have

$$
C_{2}=w
$$

For a general modulation that combines several different spatial frequencies, namely, $m(\theta)=\sum_{w} m_{w} \exp (i w \theta)$, Eq. (18) is not amenable to further simplification in the generic cases. However, we have $C_{2}=w_{0}$ in the cases that $\left|m_{w_{0}}\right|>\sum_{w \neq w_{0}}\left|m_{w}\right|$, in other words, $C_{2}$ is determined by the dominant modulation.

\section{Data availability}

All relevant data are available from the authors on request.

Received: 11 October 2016 Accepted: 26 November 2018

Published online: 19 December 2018

\section{References}

1. Lu, L., Joannopoulos, J. D. \& Soljačić, M. Topological photonics. Nat. Photonics 8, 821-829 (2014).

2. Lu, L., Joannopoulos, J. D. \& Soljačić, M. Topological states in photonic systems. Nat. Phys. 12, 626-629 (2016).

3. Khanikaev, A. B. \& Shvets, G. Two-dimensional topological photonics. Nat Photonics 11, 763 (2017).

4. Ozawa, T. et al. Topological photonics. Preprint at https://arxiv.org/abs/ 1802.04173 (2018)

5. Haldane, F. D. M. \& Raghu, S. Possible realization of directional optical waveguides in photonic crystals with broken time-reversal symmetry. Phys. Rev. Lett. 100, 013904 (2008).

6. Wang, Z., Chong, Y., Joannopoulos, J. D. \& Soljačić, M. Observation of unidirectional backscattering-immune topological electromagnetic states. Nature 461, 772-775 (2009).

7. Fu, J.-X., Liu, R.-J. \& Li, Z.-Y. Robust one-way modes in gyromagnetic photonic crystal waveguides with different interfaces. Appl. Phys. Lett. 97, 041112 (2010)
8. Poo, Y., Lin, R.-xWu,Z., Yang, Y. \& Chan, C. T. Experimental realization of self-guiding unidirectional electromagnetic edge states. Phys. Rev. Lett. 106, 93903 (2011).

9. Skirlo, S. A. et al. Experimental observation of large chern numbers in photonic crystals. Phys. Rev. Lett. 115, 253901 (2015).

10. Lu, L. et al. Experimental observation of weyl points. Science 349, 622-624 (2015).

11. Lu, L. et al. Symmetry-protected topological photonic crystal in three dimensions. Nat. Phys. 12, 337-340 (2016).

12. Slobozhanyuk, A. et al. Three-dimensional all-dielectric photonic topological insulator. Nat. Photonics 11, 130-136 (2016).

13. Armitage, N. P., Mele, E. J. \& Vishwanath, A. Weyl and dirac semimetals in three-dimensional solids. Rev. Mod. Phys. 90, 015001 (2018).

14. Li, F., Huang, X., Lu, J., Ma, J. \& Liu, Z. Weyl points and fermi arcs in a chiral phononic crystal. Nat. Phys. 14, 30 (2018).

15. Gao, W. et al. Plasmon weyl degeneracies in magnetized plasma. Nat. Commun. 7, 12435 (2016)

16. Li, F.-Y. et al. Weyl magnons in breathing pyrochlore antiferromagnets. Nat. Commun. 7, 12691 (2016).

17. Ge, H. et al. Experimental observation of acoustic weyl points and topological surface states. Phys. Rev. Appl. 10, 014017 (2018).

18. Lu, L., Fu, L., Joannopoulos, J. D. \& Soljačić, M. Weyl points and line nodes in gyroid photonic crystals. Nat. Photonics 7, 294-299 (2013).

19. Halperin, B. I. Possible states for a three-dimensional electron gas in a strong magnetic field. Jpn. J. Appl. Phys. 26, 1913 (1987).

20. Koshino, M., Aoki, H., Kuroki, K., Kagoshima, S. \& Osada, T. Hofstadter butterfly and integer quantum hall effect in three dimensions. Phys. Rev. Lett. 86, 1062 (2001).

21. Bernevig, B. A., Hughes, T. L., Raghu, S. \& Arovas, D. P. Theory of the threedimensional quantum hall effect in graphite. Phys. Rev. Lett. 99, 146804 (2007).

22. Störmer, H. L., Eisenstein, J. P., Gossard, A. C., Wiegmann, W. \& Baldwin, K. Quantization of the hall effect in an anisotropic three-dimensional electronic system. Phys. Rev. Lett. 56, 85 (1986)

23. Ran, Y., Zhang, Y. \& Vishwanath, A. One-dimensional topologically protected modes in topological insulators with lattice dislocations. Nat. Phys. 5, 298-303 (2009).

24. Jackiw, R. \& Rossi, P. Zero modes of the vortex-fermion system. Nucl. Phys. B 190, 681-691 (1981).

25. Hou, C.-Y., Chamon, C. \& Mudry, C. Electron fractionalization in twodimensional graphenelike structures. Phys. Rev. Lett. 98, 186809 (2007).

26. Iadecola, T., Schuster, T. \& Chamon, C. Non-abelian braiding of light. Phys. Rev. Lett. 117, 073901 (2016).

27. Callan, C. G. \& Harvey, J. A. Anomalies and fermion zero modes on strings and domain walls. Nucl. Phys. B 250, 427-436 (1985).

28. Wang, Z. \& Zhang, S.-C. Chiral anomaly, charge density waves, and axion strings from weyl semimetals. Phys. Rev. B 87, 161107 (2013).

29. Bi, R. \& Wang, Z. Unidirectional transport in electronic and photonic weyl materials by dirac mass engineering. Phys. Rev. B 92, 241109 (2015).

30. Schuster, T., Iadecola, T., Chamon, C., Jackiw, R. \& Pi, S.-Y. Dissipationless conductance in a topological coaxial cable. Phys. Rev. B 94, 115110 (2016).

31. EastWave V6.0., Dongjun Information and Technology Co. LTD, Shanghai, China.

32. Teo, J. C. Y. \& Kane, C. L. Topological defects and gapless modes in insulators and superconductors. Phys. Rev. B 82, 115120 (2010).

33. Qi, X.-L., Hughes, T. L. \& Zhang, S.-C. Topological field theory of timereversal invariant insulators. Phys. Rev. B 78, 195424 (2008).

34. Zhang, S.-C. \& Hu, J. A four-dimensional generalization of the quantum hall effect. Science 294, 823-828 (2001).

35. Kraus, Y. E., Ringel, Z. \& Zilberberg, O. Four-dimensional quantum hall effect in a two-dimensional quasicrystal. Phys. Rev. Lett. 111, 226401 (2013).

36. Price, H. M., Zilberberg, O., Ozawa, T., Carusotto, I. \& Goldman, N. Fourdimensional quantum hall effect with ultracold atoms. Phys. Rev. Lett. 115, 195303 (2015).

37. Ozawa, T., Price, H. M., Goldman, N., Zilberberg, O. \& Carusotto, I. Synthetic dimensions in integrated photonics: from optical isolation to fourdimensional quantum hall physics. Phys. Rev. A. 93, 043827 (2016).

38. Sugawa, S., Salces-Carcoba, F., Perry, A. R., Yue, Y. \& Spielman, I. B. Second chern number of a quantum-simulated non-abelian yang monopole. Science 360, 1429-1434 (2018).

39. Lohse, M., Schweizer, C., Price, H. M., Zilberberg, O. \& Bloch, I. Exploring 4d quantum hall physics with a $2 \mathrm{~d}$ topological charge pump. Nature 553, 55 (2018).

40. Zilberberg, O. et al. Photonic topological boundary pumping as a probe of $4 \mathrm{~d}$ quantum hall physics. Nature 553, 59 (2018).

41. Li, F.-F. et al. Topological light-trapping on a dislocation. Nat. Commun. 9, $2462(2018)$ 
42. Sun, L., Jiang, S. \& Marciante, J. R. Compact all-fiber optical faraday components using 65-wt\%-terbium-doped fiber with a record verdet constant of- $32 \mathrm{rad} /(\mathrm{tm})$. Opt. Express 18, 12191-12196 (2010).

43. Schmidt, M. A. et al. Complex faraday rotation in microstructured magnetooptical fiber waveguides. Adv. Mater. 23, 2681-2688 (2011).

44. Onbasli, M. C. et al. Optical and magneto-optical behavior of cerium yttrium iron garnet thin films at wavelengths of 200-1770 nm. Sci. Rep. 6, 23640 (2016).

45. Luo, X., Zhou, M., Liu, J., Qiu, T. \& Yu, Z. Magneto-optical metamaterials with extraordinarily strong magneto-optical effect. Appl. Phys. Lett. 108, 131104 (2016).

46. Kang, M. S., Butsch, A. \& Russell, P. St. J. Reconfigurable light-driven optoacoustic isolators in photonic crystal fibre. Nat. Photonics 5, 549-553 (2011).

47. Hur, K. et al. Three-dimensionally isotropic negative refractive index materials from block copolymer self-assembled chiral gyroid networks. Angew. Chem. Int. Ed. 50, 11985-11989 (2011).

48. Khoo, I. C., Hong, K. L., Zhao, S., Ma, D. \& Lin, T.-H. Blue-phase liquid crystal cored optical fiber array with photonic bandgaps and nonlinear transmission properties. Opt. Express 21, 4319-4328 (2013).

49. Turner, M. D. et al. Miniature chiral beamsplitter based on gyroid photonic crystals. Nat. Photonics 7, 801-805 (2013).

50. Ullal, C. K. et al. Triply periodic bicontinuous structures through interference lithography: a level-set approach. JOSA A 20, 948-954 (2003).

51. Kopp, V. I. \& Genack, A. Z. Chiral fibres: adding twist. Nat. Photonics 5, 470-472 (2011).

52. Wong, G. K. L. et al. Excitation of orbital angular momentum resonances in helically twisted photonic crystal fiber. Science 337, 446-449 (2012).

53. Bi, R., Yan, Z., Lu, L. \& Wang, Z. Topological defects in floquet systems: anomalous chiral modes and topological invariant. Phys. Rev. B 95, 161115 (2017).

54. Yuan, L., Lin, Q., Xiao, M. \& Fan, S. Synthetic dimension in photonics. Optica 5, 1396-1405 (2018)

55. Xie, K. et al. Fiber guiding at the dirac frequency beyond photonic bandgaps. Light.: Sci. \& Appl. 4, e304 (2015).

56. Markos, C., Travers, J. C., Abdolvand, A., Eggleton, B. J. \& Bang, O. Hybrid photonic-crystal fiber. Rev. Mod. Phys. 89, 045003 (2017).

57. Bulgakov, E. \& Sadreev, A. Fibers based on propagating bound states in the continuum. Phys. Rev. B 98, 085301 (2018).

\section{Acknowledgements}

We thank the discussion with Jian Wang, Wei Ding, and Changyuan Yu on fiber technologies and with Hannah M. Price and Chen Fang on 3D QHE. L.L. was supported by the National key R\&D Program of China under grant no. 2017YFA0303800, 2016YFA0302400, and by NSFC under project no. 11721404. Z.W. was supported by NSFC under grant no. 11674189.

\section{Author contributions}

L.L. and Z.W. initiated the project and prepared the manuscript. L.L. computed the band structures, H.G. performed the time-domain simulations, and Z.W. obtained the analytical solutions.

\section{Additional information}

Supplementary Information accompanies this paper at https://doi.org/10.1038/s41467018-07817-3.

Competing interests: The authors declare no competing interests.

Reprints and permission information is available online at http://npg.nature.com/ reprintsandpermissions/

Publisher's note: Springer Nature remains neutral with regard to jurisdictional claims in published maps and institutional affiliations.

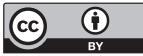

Open Access This article is licensed under a Creative Commons Attribution 4.0 International License, which permits use, sharing, adaptation, distribution and reproduction in any medium or format, as long as you give appropriate credit to the original author(s) and the source, provide a link to the Creative Commons license, and indicate if changes were made. The images or other third party material in this article are included in the article's Creative Commons license, unless indicated otherwise in a credit line to the material. If material is not included in the article's Creative Commons license and your intended use is not permitted by statutory regulation or exceeds the permitted use, you will need to obtain permission directly from the copyright holder. To view a copy of this license, visit http://creativecommons.org/ licenses/by/4.0/.

(C) The Author(s) 2018 